\title{
A time domain BEM for transient dynamic straight crack in piezoelectric solids
}

\author{
Peng Zhao ${ }^{2}$ \& Taiyan Qin ${ }^{1}$ \\ ${ }^{1}$ College of Science, China Agricultural University, China \\ ${ }^{2}$ College of Engineering, China Agricultural University, China
}

\begin{abstract}
A singular time-domain boundary element method for transient elastodynamic crack analysis in two-dimensional piezoelectric solids is presented in this paper. A finite straight crack in infinite piezoelectric solids under impacted loading is investigated. A convolution quadrature formula is applied for temporal discretization, while the Gauss-Chebyshev method is adopted for the spatial integration. By use of Laplace transform, time domain Green's functions for the infinite plane are split into singular plus regular terms, the singular ones coinciding with the static Green's function. Numerical examples are presented to show the accuracy of this method, and then the contribution of the piezoelectric effect on the variation of dynamic stress intensity factors is discussed.
\end{abstract}

Keywords: piezoelectric solids, dynamic crack analysis, dynamic intensity factors.

\section{Introduction}

Due to coupling effects between the mechanical and the electrical fields, piezoelectric materials are widely applied in transducers, actuators, and many other smart devices and structures. Dynamic crack analysis in piezoelectric solids is an important issue in fracture and damage mechanics as well as nondestructive testing. It is very useful to characterize and evaluate the mechanical and the electrical integrity, the reliability and the durability of piezoelectric devices and structures. As to the complexity of the corresponding initial-boundary value problems, many numerical methods have been applied. Enderlein [1] and Enderlein et al: [2] have applied the finite element method for 2-D dynamic crack analysis in piezoelectric solids. Dziatkiewicz and Fedelinski 
[3], Gaul et al. [4] have developed dual reciprocity BEM to avoid the difficulty to get Green's functions. Time-domain BEM for transient dynamic crack analysis in piezoelectric solids has been present in [5-9]. Meshless methods for piezoelectric solids have been implemented by Liu et al. [10] and Sladek et al: [11].

For temporal discretization, the quadrature formula of Lubich $[12,13]$ is adopted for approximating the Riemann convolution integrals. For spatial integral, Gauss-Chebyshev method is implemented. A special feature of present time-domain BEM is that it requires only Laplace-domain instead of timedomain dynamic piezoelectric fundamental functions. Cauchy principal boundary integrals arising in the present time-domain BEM are computed by using Gauss-Chebyshev method. A basic function is multiplied, to describe the local behavior of the displacement density functions properly.

\section{Basic equations}

A homogenous and linear piezoelectric solid containing a finite crack is considered. In the absence of body forces and under the quasi-electric assumption, the cracked solid satisfies the equations of motion and the Gauss's law [14]

$$
\begin{gathered}
\sigma_{i j, j}=\rho \ddot{u_{i}}, \\
D_{i, i}=0
\end{gathered}
$$

where $u_{i}$ and $\sigma_{i j}$ represent the displacement and the stress components, $\rho$ is the mass density, and $D_{i}$ denotes the electric displacements, a comma represents spatial derivatives, and superscript denote temporal derivatives. The piezoelectric solid satisfies the following constitutive equations

$$
\begin{gathered}
\sigma_{i j}=c_{i j k l} u_{k, l}-e_{i j k} E_{k} \\
D_{i}=e_{k l i} u_{k, l}+\varepsilon_{i k} E_{k}
\end{gathered}
$$

in which $c_{i j k l}$ is elasticity tensor, $e_{i j k}$ is the piezoelectric tensor, $\varepsilon_{i k}$ is the dielectric permittivity tensor, and $E_{k}$ represents the components of electrical field. Under quasi-electrostatic assumption, the electrical field components $E_{k}$ and the electrical potential $\phi$ are related by

$$
E_{k}=-\phi_{, k}
$$

For convenience, the generalized displacements, the generalized stresses, and the generalized elasticity tensor are introduced as follows

$$
U_{I}= \begin{cases}u_{I}, & I=1,2 \\ \phi, & I=3\end{cases}
$$




$$
\begin{gathered}
\Sigma_{i J}=\left\{\begin{array}{lc}
\sigma_{i J}, & J=1,2 \\
D_{i}, & J=3
\end{array}\right. \\
C_{i J K l}= \begin{cases}c_{i J K l}, & J, K=1,2 \\
e_{K l i}, & J=3, K=1,2 \\
e_{J i l}, & K=3, J=1,2 \\
-\varepsilon_{i l}, & J=K=3\end{cases}
\end{gathered}
$$

Thus, the equations of motion and constitutive equations can be rewritten as

$$
\begin{aligned}
& \Sigma_{i J, i}=\rho \delta_{J K}^{*} \ddot{U}_{K} \\
& \Sigma_{i J}=C_{i J K l} U_{K, l}
\end{aligned}
$$

In Eq. (9), $\delta_{J K}^{*}$ is the generalized Kronecker delta defined by

$$
\delta_{J K}^{*}= \begin{cases}\delta_{J K}, & J, K=1,2 \\ 0, & \text { otherwise }\end{cases}
$$

\section{Integral equations}

An infinite piezoelectric solid containing a finite crack as shown in Fig. 1 is considered, that the length of crack is $2 a$ and axis $x_{2}$ is parallel with polarization direction.
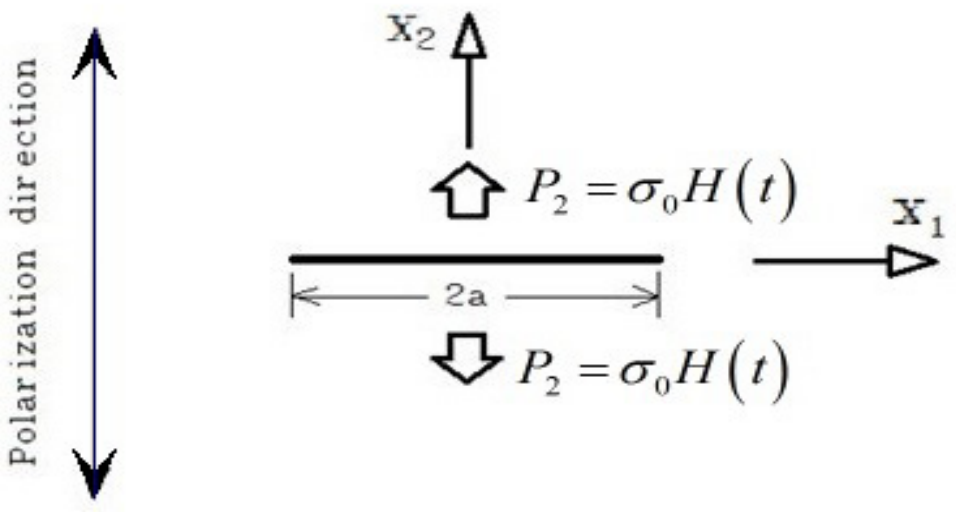

Figure 1: $\quad$ A straight crack under impact mechanical loading.

Initial conditions and boundary conditions are assumed

$$
U_{I}(\mathrm{x}, t)=\dot{U}_{I}(\mathrm{x}, t)=0, \quad \text { for } \quad \mathrm{t}=0,
$$




$$
P_{I}(\mathrm{x}, t)=P_{I}^{*}(\mathrm{x}) H(t), \quad \text { for } \quad \mathrm{x} \in \Gamma_{C},
$$

where the $P_{I}^{*}(\mathrm{x})$ represents the amplitude of the impact loading, $H(t)$ denotes the Heaviside step function.

By using the generalized Betti-Rayleigh reciprocity theorem, the formula for the extended displacements can be obtained

$$
U_{J}(\mathrm{x}, t)=-\int_{\Gamma_{c^{+}}} T_{I J}^{G}(\mathrm{x}, \mathrm{y}, t) * \tilde{U}_{I}(\mathrm{y}, t) \mathrm{d} \Gamma_{\mathrm{y}},
$$

where $T_{I J}^{G}(\mathrm{x}, \mathrm{y}, t)$ are traction fundamental solutions, $\mathrm{x}$ and $\mathrm{y}$ represent the source and the observation points, asterisk ' $*$ ' denotes the Riemann convolution. By substituting the Eq. (12) into the constitutive relations Eq. (4), taking the limit process $\mathrm{X} \rightarrow \Gamma_{C^{+}}$, and considering the boundary conditions Eq. (13), time domain traction BIEs can be obtained

$$
\text { f.p. } \int_{\Gamma_{c^{+}}}-n_{i}(\mathrm{x}) C_{i J K l} \frac{\partial T_{K M}^{G}(\mathrm{x}, \mathrm{y}, t)}{\partial x_{l}} * \tilde{U}_{M}(\mathrm{y}, t) \mathrm{d} \Gamma_{\mathrm{y}}=P_{J}(\mathrm{x}, t)
$$

where f.p. denotes the hypersingular Hadamard finite-part integral, and generalized displacement discontinuous functions $\tilde{U}_{M}(\mathrm{y}, t)$ are defined by

$$
\tilde{U}_{M}(\mathrm{y}, t)=U_{M}\left(\mathrm{y} \in \Gamma_{C^{+}}, t\right)-U_{M}\left(\mathrm{y} \in \Gamma_{C^{-}}, t\right) .
$$

Using the relations

$$
\begin{gathered}
\frac{\partial T_{I J}^{G}(\mathrm{x}, \mathrm{y}, t)}{\partial x_{1}}=-\frac{\partial T_{I J}^{G}(\mathrm{x}, \mathrm{y}, t)}{\partial y_{1}}, \\
\frac{\partial T_{I J}^{G}(\mathrm{x}, \mathrm{y}, t)}{\partial x_{2}}=-\frac{\partial \sigma_{1 I J}^{G}(\mathrm{x}, \mathrm{y}, t)}{\partial y_{1}}+\rho \delta_{I K}^{*} \ddot{U}_{K J}^{G},
\end{gathered}
$$

and integration by parts, the hypersingular traction integral Equations (15) can be degenerated into Cauchy integral equations

$$
\begin{aligned}
n_{i}(\mathrm{x}) \int_{\Gamma_{c^{+}}}( & C_{i J K 1} T_{K M}^{G}(\mathrm{x}, \mathrm{y}, t)+C_{i J K 2} \sigma_{1 K M}^{G}(\mathrm{x}, \mathrm{y}, t)+ \\
& \left.C_{i J K 2} \int_{y_{1}}^{a} \rho \delta_{K P}^{*} \ddot{U}_{P M}^{G} \mathrm{~d} \Gamma_{\mathrm{y}}\right) * f_{M}(\mathrm{y}, t) \mathrm{d} \Gamma_{\mathrm{y}}=P_{J}(\mathrm{x}, t),
\end{aligned}
$$

where $f_{M}(\mathrm{y}, t)=\partial \tilde{U}_{M}(\mathrm{y}, t) / \partial y_{1}$ is the basic unknown function, and $\sigma_{1 K M}^{G}(\mathrm{x}, \mathrm{y}, t)$ is defined

$$
\sigma_{1 K M}^{G}(\mathrm{x}, \mathrm{y}, t)=\left.T_{I J}^{G}(\mathrm{x}, \mathrm{y}, t)\right|_{n(y)=(1,0)} \quad .
$$




\section{Temporal and spatial procedure}

\subsection{Time discretization}

To solve the Riemann convolution, the convolution quadrature formula of Lubich $[12,13]$ is applied for temporal discretization. As the convolution quadrature formula of Lubich, the integral equations (19) are turned into

$$
n_{i}(\mathrm{x}) \int_{\Gamma_{c^{+}}} \sum_{m=0}^{M} \omega(x, y, m \Delta t) \times \frac{\partial \tilde{U}_{M}(\mathrm{y},(M-m) \Delta t)}{\partial y_{1}} \mathrm{~d} \Gamma_{\mathrm{y}}=P_{J}(\mathrm{x}, M \Delta t)
$$

where the time $t$ is divided into $M$ equal time-steps $\Delta t$, and the weights $\omega(x, y, m \Delta t)$ are determined by

$$
\begin{gathered}
\omega(x, y, m \Delta t)=\frac{r^{-m}}{Q} \sum_{q=0}^{Q-1}\left(C_{i J K 1} \widehat{T}_{K M}^{G}\left(\mathrm{x}, \mathrm{y}, s_{q}\right)+C_{i J K 2} \widehat{\sigma}_{1 K M}^{G}\left(\mathrm{x}, \mathrm{y}, s_{q}\right)\right. \\
\left.+C_{i J K 2} \int_{y_{1}}^{b} \rho s_{q}^{2} \delta_{K P}^{*} \hat{U}_{P M}^{G} \mathrm{~d} \Gamma_{\mathrm{y}}\right) e^{-2 \pi i \cdot m q / Q}
\end{gathered}
$$

in which $\hat{T}, \hat{\sigma}, \hat{U}$ are Laplace transform of the function $T, \sigma, U$, and

$$
s_{q}=\delta\left(\zeta_{q}\right) / \Delta t, \delta\left(\zeta_{q}\right)=\sum_{j=1}^{2} \frac{\left(1-\zeta_{q}\right)^{j}}{2}, \zeta_{q}=r e^{i \cdot 2 \pi q / Q}, r=\varepsilon^{1 /(2 Q)}
$$

with $\varepsilon$ being the numerical error in computing the Laplace transform.

\subsection{Spatial approximate}

Time-domain and Laplace-domain dynamic fundamental solutions for homogenous and linear piezoelectric solids have been derived in $[15,16]$. Unfortunately, they cannot be given in closed forms, but they can be represented by line-integrals over a unit-circle in 2-D case. Though time-domain BEM formulation presented in this paper, only the Laplace-domain fundamental solutions are needed in temporal discretization. Note here that the tractions BIEs are Cauchy singular and should be understood in sense of Cauchy-principal value integrals. 2-D Laplace-domain displacement fundamental solution can be expressed as $[15,16]$

$$
\hat{U}_{I J}(\mathbf{x}, \mathbf{y}, s)=\frac{1}{8 \pi^{2}} \int_{|\eta|=1} \sum_{m=1}^{2} \frac{\gamma_{I J}^{m}}{\rho c_{m}^{2}} \Psi\left(s|\boldsymbol{\eta} \bullet(\mathbf{y}-\mathbf{x})| / c_{m}\right) \mathrm{d} S_{\eta}
$$

where $\gamma_{I J}^{m}$ and $\rho c_{m}^{2}$ are determined by wave propagation vector $\boldsymbol{\eta}=\left(\eta_{1}, \eta_{2}\right)$ and material constants, and $s$ is the Laplace-transform parameter. The function $\Psi(z)$ is defined by

$$
\Psi(z)=-\left[e^{-z} \operatorname{Ei}[z]+e^{z} \operatorname{Ei}(-z)\right]
$$


with $\operatorname{Ei}[z]$ being the exponential integral and $z$ a complex variable. The function $\Psi(z)$ has a logarithmic singularity when integration is taken over the collocation point.

The displacement fundamental solution can be decomposed into regular and singular static parts as

$$
\hat{U}_{I J}(\mathbf{x}, \mathbf{y}, s)=\hat{U}_{I J}^{R}(\mathbf{x}, \mathbf{y}, s)+\hat{U}_{I J}^{S}(\mathbf{x}, \mathbf{y})
$$

where the regular dynamic part is given by

$$
\begin{aligned}
& \hat{U}_{I J}^{R}(\mathbf{x}, \mathbf{y}, s)=\frac{1}{8 \pi^{2}} \int_{|\eta|=1} \sum_{m=1}^{2} \frac{\gamma_{I J}^{m}}{\rho c_{m}^{2}} \Psi^{R}\left(s|\boldsymbol{\eta} \cdot(\mathbf{y}-\mathbf{x})| / c_{m}\right) \mathrm{d} S_{\eta}, \\
& \Psi^{R}(a, b)=\Psi(a b)+2 \log b
\end{aligned}
$$

while the singular static part has the following form

$$
\hat{U}_{I J}^{S}(\mathbf{x}, \mathbf{y})=\frac{1}{8 \pi^{2}} \int_{|\eta|=1} \sum_{m=1}^{2} \frac{\gamma_{I J}^{m}}{\rho c_{m}^{2}} \log (|\boldsymbol{\eta} \bullet(\mathbf{y}-\mathbf{x})|) \mathrm{d} S_{\eta}
$$

The static displacement fundamental solution can be reduced to an explicit form as [17]

$$
\hat{U}_{I J}^{S}(\mathbf{x}, \mathbf{y})=-\frac{1}{\pi} \operatorname{Re}\left[\sum_{M=1}^{3} P_{J M} Q_{M I} \ln \left(z_{M}-z_{M}^{0}\right)-\ln \left(i+\mu_{M}\right)\right]
$$

where

$$
z_{M}=y_{1}+\mu_{M} y_{2}, \quad z_{M}^{0}=x_{1}+\mu_{M} x_{2}, \quad M=1,2,3
$$

are the counterpart of the collocation and the integration point, $\mu_{M}, P_{J M}$ and $Q_{M I}$ are determined by material constants.

So, the corresponding traction fundamental solutions can be obtained as

$$
\begin{gathered}
\hat{T}_{I J}^{R}(\mathbf{x}, \mathbf{y}, s)=\frac{1}{8 \pi^{2}} \int_{|\eta|=1} \sum_{m=1}^{2} \frac{R_{I J}^{m}}{\rho c_{m}^{2}} \cdot \frac{s}{c_{m}} \cdot \Psi^{\prime}\left(s|\boldsymbol{\eta} \bullet(\mathbf{y}-\mathbf{x})| / c_{m}\right) \\
\times \operatorname{sign}[\boldsymbol{\eta} \bullet(\mathbf{y}-\mathbf{x})] \mathrm{d} S_{\eta}, \\
\hat{T}_{I J}^{S}(\mathbf{x}, \mathbf{y}, s)=\frac{1}{\pi} \operatorname{Re}\left[\sum_{M}^{3} L_{J M} Q_{M I} \frac{\mu_{M} n_{1}(\mathbf{y})-n_{2}(\mathbf{y})}{z_{M}-z_{M}^{0}}\right], \\
\hat{\sigma}_{1 I J}^{R}(\mathbf{x}, \mathbf{y}, s)=\frac{1}{8 \pi^{2}} \int_{|\eta|=1} \sum_{m=1}^{2} \frac{C_{1 I K l} \gamma_{J K}^{m} \eta_{l}}{\rho c_{m}^{2}} \cdot \frac{s}{c_{m}} \cdot \Psi^{\prime}\left(s|\boldsymbol{\eta} \cdot(\mathbf{y}-\mathbf{x})| / c_{m}\right) \\
\times \operatorname{sign}[\mathbf{\eta} \bullet(\mathbf{y}-\mathbf{x})] \mathrm{d} S_{\eta}, \\
\hat{\sigma}_{1 I J}^{S}(\mathbf{x}, \mathbf{y})=\frac{1}{\pi} \operatorname{Re}\left[\sum_{M=1}^{3} L_{J M} Q_{M I} \frac{\mu_{M}}{z_{M}-z_{M}^{0}}\right],
\end{gathered}
$$


where $L_{J M}$ is also determined by material constants. More details of fundamental solutions can be found in García-Sánchez et al. [9].

By substituting Eqs (31)-(34) into Eqs (22), and after normalized the limits of integration, the problem can be rewritten as

$$
\begin{array}{r}
\sum_{j=0}^{N}\left(\int_{-1}^{1} \alpha_{i J}^{M} \frac{f_{M}(\xi, j \Delta t)}{\xi-\eta}+\beta_{i J}^{M}(\xi, \eta,(N-j) \Delta t) f_{M}(\xi, j \Delta t) \mathrm{d} \xi\right) \\
=P_{J}(\eta, N \Delta t)
\end{array}
$$

where $\alpha_{i J}^{M}$ are determined by material constants, $\beta_{i J}^{M}(\xi, \eta,(N-j) \Delta t)$ are the regular parts of integral kernel. Dealing with the Cauchy integration, GaussChebyshev method is applied. The $f_{M}(\xi, t)$ can be approximated by a series Chebyshev polynomial multiplied a weight function as

$$
f_{M}(\xi, t)=w(\xi) F_{M}(\xi, t),
$$

where $w(\xi)$ is the weight function, and the function $F_{M}(\xi, t)$ are unknown bounded functions

$$
w(\xi)=(1-\xi)^{-1 / 2}, \quad F_{M}(\xi, t)=\sum_{n=1}^{\infty} C_{M}(t) T_{n}(\xi) .
$$

By using the relation of Chebyshev polynomial [18]

$$
\frac{1}{\pi} \int_{-1}^{1} \frac{\phi(\xi)}{\xi-\eta_{j}} \mathrm{~d} \xi=\frac{1}{\pi} \int_{-1}^{1} \sum_{i=1}^{n} \frac{\left(1-\xi^{2}\right)^{1 / 2} T_{n}(\xi)}{\xi-\eta_{j}} \mathrm{~d} \xi=\sum_{i=1}^{n} \frac{F\left(\xi_{i}\right)}{n\left(\xi_{i}-\eta_{j}\right)}
$$

where

$$
\xi_{i}=\cos \left[\frac{(2 i-1) \pi}{2 n}\right], i=1, \cdots, n ; \quad \eta_{j}=\cos \left[\frac{j}{n} \pi\right], j=1, \cdots, n-1 .
$$

The integral Eqs (35) are turned into the following linear algebraic equations

$$
\begin{array}{r}
\sum_{j=0}^{N}\left(\sum_{i=1}^{n} \frac{\pi}{n} F_{M}\left(\xi_{i}, j \Delta t\right)\left[\frac{\alpha_{M}}{\xi_{i}-\eta_{k}}+\beta_{M}\left(\xi_{i}, \eta_{k},(N-j) \Delta t\right)\right]\right) \\
=P_{J}\left(\eta_{k}, N \Delta t\right) .
\end{array}
$$

To solve the equations, supplementary equations are needed. Duo to displacement discontinuous functions at the crack tip are zero, the supplementary equations are

$$
\int_{-1}^{1} \partial \tilde{U}_{M}(\xi, j \Delta t) / \partial \xi \mathrm{d} \xi=\int_{-1}^{1} f_{M}(\xi, j \Delta t) \mathrm{d} \xi=0
$$

Using the same scheme, the integral equations can be reduced as

$$
\sum_{i=1}^{n} \frac{\pi}{n} F_{M}\left(\xi_{i}, j \Delta t\right)=0
$$

Solving Eqs (40) and (42), the unknown functions $F_{M}\left(\xi_{i}, t\right)$ can be computed, then by interpolating, $f_{M}(\xi, t)$ can be obtained. 


\subsection{Computation of dynamic intensity factors}

Since $F_{M}\left(\xi_{i}, j \Delta t\right)$ has been solved, the value of $F_{M}(\xi, t)$ at the crack tips can be approximated by Lagrange extrapolation. The generalized stress intensity factors $K$ can be computed as

$$
\begin{aligned}
& K_{\mathrm{I}}(j \Delta t)=\lim _{r \rightarrow 0} \sqrt{2 r} \Sigma_{22}=\sqrt{2} \sum_{M=1}^{3} \alpha_{12}^{M} F_{M}(1, j \Delta t), \\
& K_{\mathrm{II}}(j \Delta t)=\lim _{r \rightarrow 0} \sqrt{2 r} \Sigma_{12}=\sqrt{2} \sum_{M=1}^{3} \alpha_{22}^{M} F_{M}(1, j \Delta t), \\
& K_{\mathrm{IV}}(j \Delta t)=\lim _{r \rightarrow 0} \sqrt{2 r} \Sigma_{23}=\sqrt{2} \sum_{M=1}^{3} \alpha_{23}^{M} F_{M}(1, j \Delta t),
\end{aligned}
$$

where $\alpha_{i j}^{M}$ are determined by material constants.

\section{Numerical examples}

\subsection{Impact mechanical loading}

The intensity factors are normalized as

$$
K_{\mathrm{I}}^{*}(t)=\frac{K_{\mathrm{I}}(t)}{K_{\mathrm{I}}^{s t}}, \quad K_{\mathrm{IV}}^{*}=\frac{K_{\mathrm{IV}}(t)}{K_{\mathrm{I}}^{s t}},
$$

where $K_{\mathrm{I}}^{s t}=\sigma_{0} \sqrt{a}$.

To examine this method's accuracy, numerical calculations are carried out for piezoelectric material PZT-5H, whose constants are given in Table 1. The finite crack is subjected to an impact mechanical loading $P_{2}(\mathrm{x}, t)=P_{2}^{*}(\mathrm{x}) H(t)$. The numerical results for $K_{\mathrm{I}}^{*}(t)$ and $K_{\mathrm{IV}}^{*}(t)$ have been given in Figs 2 and 3 versus the time dimensionless time $t c_{T} / a$, where $c_{T}=\sqrt{C_{66} / \rho}$. Comparing the results with that obtained by García-Sánchez et al. [6] a good agreement between both results is shown.

Table 1: $\quad$ Material properties used in this work.

\begin{tabular}{|c|c|c|c|c|c|c|c|c|c|c|}
\hline & $C_{11}$ & $C_{12}$ & $C_{22}$ & $C_{66}$ & $e_{21}$ & $e_{22}$ & $e_{16}$ & $\varepsilon_{11}$ & $\varepsilon_{22}$ & $\rho$ \\
\hline PZT-5H & 126 & 84.1 & 117 & 23 & -6.5 & 23.3 & 17.0 & 15.04 & 13.0 & 7500 \\
\hline PZT-6B & 168 & 60 & 163 & 27.1 & -0.9 & 7.1 & 4.6 & 3.6 & 3.4 & 7600 \\
\hline BaTiO $_{3}$ & 150 & 66 & 146 & 44 & -4.35 & 17.5 & 11.4 & 9.87 & 11.2 & 5800 \\
\hline
\end{tabular}




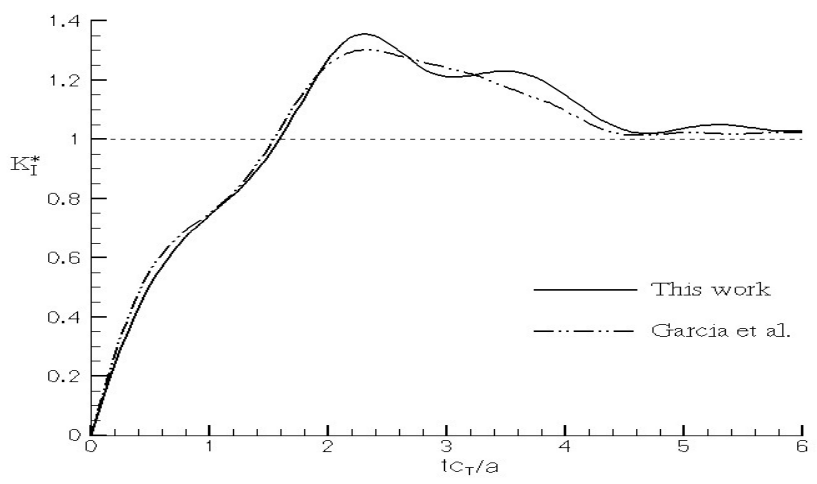

Figure 2: $\quad$ Normalized mode-I stress intensity factor versus time for impact mechanical loading.

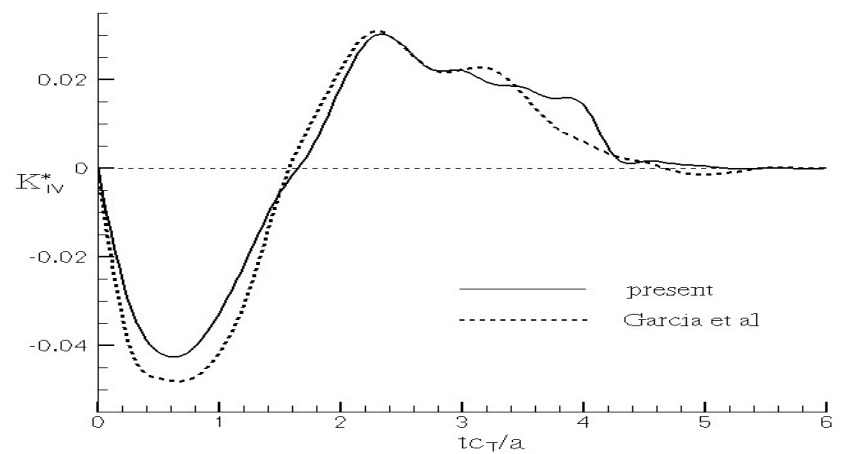

Figure 3: Normalized electrical displacement intensity factor versus time for impact mechanical loading.

\subsection{Infuluence of piezoelectric effect}

As we know that the electric field doesn't contribute to $\mathrm{K}_{\mathrm{I}}$ under static loading, but the electric field does contribute the changes over time of $\mathrm{K}_{\mathrm{I}}$. In order to find out the influence of piezoelectric effect, numerical caculations are carried out for two different typical piezoelectric materials PZT-6B and $\mathrm{BaTiO}_{3}$. Firstly, we obtain the dynamic stress intensity KI of them. Then we let the piezoelectric tensor to be zero and obtain the $\mathrm{K}_{\mathrm{I}}$ as well, that means piezoelectric effect does not take into account in this case. Corresponding results for the normalized dynamic intensity factors are presented in Figs 4 and 5.

It shows that stress intensity factor $\mathrm{K}_{\mathrm{I}}$ is drastically affected by piezoelectric effect. Without considering the piezoelectric effect, $K_{\mathrm{I}}^{*}(t)$ is larger at the beginning, and reaches the maximun value earlier, but the peak value is much smaller. 


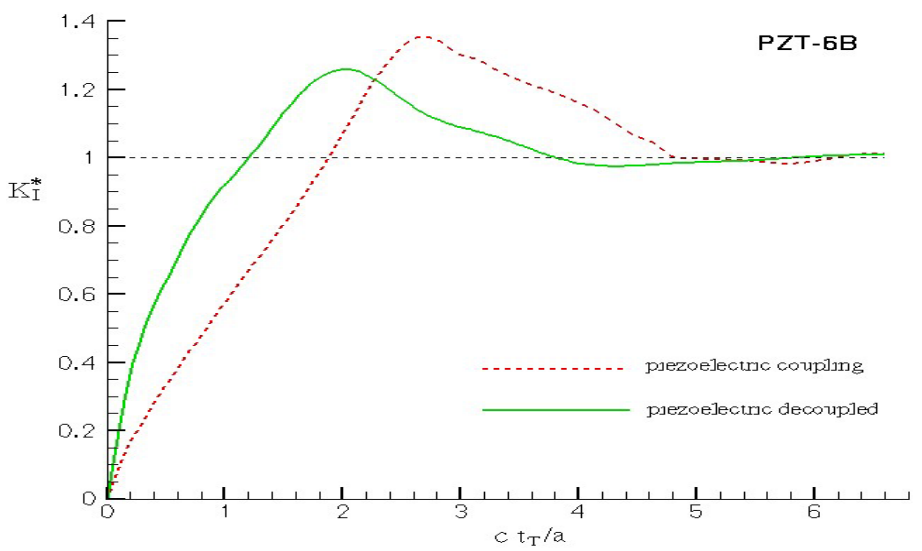

Figure 4: Normalized mode-I stress intensity factor versus time for impact mechanical loading.

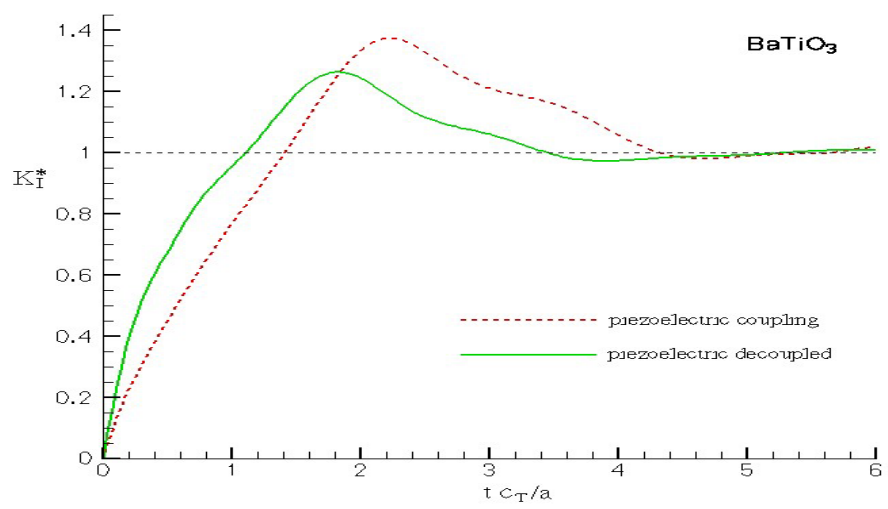

Figure 5: Normalized mode-I stress intensity factor versus time for impact mechanical loading.

\section{Conclusions}

Transient dynamic crack analysis in two-dimensional, homogenous and linear piezoelectric solids is presented in this paper. A Cauchy singular time-domain traction BEM is developed for this purpose. By using Lubich quadrature formula, Laplace-domain instead of time-domain dynamic fundamental solutions are applied, which is much more stable. By using the properties of straight crack and relations of fundamental solutions, the hypersingular integral equations are converts to Cauchy singular equations. Then, the singular integrals are treated by Gauss-Chebyshev method. Since the weight functions fully reflect the crack front singularity, dynamic intensity factors can be computed directly from the Displacement Discontinuity density functions, which is very accurate and easy. 
Discussion on the influence of piezoelectric effect, the results presented here are useful to gain a better understanding of piezoelectric effect under impact loading conditions. It shows that in dealing with dynamic problems, piezoelectric effect must be considered, which has significant influence.

\section{Acknowledgement}

The project was supported by the National Natural Science Foundation of China (No. 11172320), which is gratefully acknowledged.

\section{References}

[1] M. Enderlein, FEM-analysis of cracks in piezoelectric structures under dynamic electromechanical loading, in: F. Nilsson (Ed.), Proceedings of the 15th European Conference on Fracture, Stockholm, Sweden, 2004.

[2] M. Enderlein, A. Ricoeur, M. Kuna, Finite element techniques for dynamic crack analysis in piezoelectrics, Int. J. Fract. 134, pp. 191-208, 2005.

[3] G. Dziatkiewicz, P. Fedelinski, Dynamic analysis of piezoelectric structures by the dual reciprocity boundary element method, in: B. Gatmiri, A. Sellier, M. H Aliabadi, (Eds.), Advances in Boundary Element Techniques VII. EC Ltd., UK, pp. 121-126, 2006.

[4] L. Gaul, M. Kögl, M. Wagner, Boundary Element Methods for Engineers and Scientists, Springer, Berlin, 2003.

[5] F. García-Sánchez, Ch. Zhang, A. Sáez, A 2-D time-domain BEM for dynamic analysis of cracked piezoelectric solids, in: B. Gatmiri, A. Sellier, M.H. Aliabadi (Eds.), Advances in Boundary Element Techniques VII.. EC Ltd., UK, pp. 165-171, 2006.

[6] F. García-Sánchez, Ch. Zhang, J. Sladek, V. Sladek, 2-D transient dynamic crack analysis in piezoelectric solids by BEM, Comput. Mater. Sci. 39, pp. 179-186, 2007.

[7] Ch. Zhang, Transient dynamic analysis of cracked piezoelectric solids by time-domain BIEM, in: H.A. Mang, F.G. Rammerstorfer, J. Eberhardsteiner (Eds.), Proceedings of the Fifth Word Congress on Computational Mechanics (WCCMV), July 7-12, 2002. Vienna University of Technology, Vienna, Austria-ISBN 3-9501554-0-6.

[8] Ch. Zhang, C.-Y. Wang, S. Hirose, A time-domain BIEM for dynamic crack analysis of a piezoelectric solid, in: Z. Yao, M.H. Alisbadi (Eds.), Boundary Element Techniques, Tsinghua University Press, Beijing, China. pp. 119-126, 2002.

[9] F. García-Sánchez, Ch. Zhang, A. Sáez, 2-D transient dynamic analysis of cracked piezoelectric solids by a time-domain BEM, Comput. Methods Appl. Mech. Engrg. 197, pp. 3108-3121, 2008.

[10] G.R. Liu, K.Y. Dai, K.M. Lim, Y.T. Gu, A point interpolation mesh free method for static and frequency analysis of two-dimensional piezoelectric structures, Comput. Mesh. 29, pp. 510-590, 2002. 
[11] J. Sladek, V. Sladek, Ch. Zhang, F. García-Sánchez, M Wüunche, Meshless local Petrov-Galerkin method for plane piezoelectricity, Comput. Mater. Continua. 4, pp. 109-118, 2002.

[12] C. Lubich, Convolution Quadrature and Discretized Operational Calculus I. Numer. Math. 52, pp. 129-145, 1988.

[13] C. Lubich, Convolution Quadrature and Discretized Operational Calculus II. Numer. Math. 52, pp. 413-425, 1988.

[14] W.G. Cady, Piezoelectricity, Dover Publishers, New York, 1964.

[15] C.-Y. Wang, Ch. Zhang, 2D and 3D dynamic Green's functions and timedomain BIE formulations for piezoelectric solids, in: Z.H. Yao, M.W. Yuan, W.X. Zhong (Eds.), Proceedings of the WCCM VI in Conjunction with APCOM'04, September 5-10, 2004, Tsinghua University Press and Springer Verlag, Beijing, China, 2004.

[16] C.Y. Wang, Ch. Zhang, 3-D and 2-D dynamic Green's functions and timedomain BIEs for piezoelectric solids, Engng. Anal. Bound. Elem. 29, pp. 454-465, 2005.

[17] E. Pan, A BEM analysis of fracture mechanics in 2-D anisotropic piezoelectric solids, Engng. Anal. Bound. Elem. 23, pp. 67-76, 1999.

[18] A.C. Kaya, F. Erdogan, On the solution of integral equations with strongly singular kernels, Quart. App. Math. April. pp. 105-122, 1987. 